\title{
Oral Rehabilitation of a Patient with Cerebellopontine Angle Epidermoid Cyst
}

Amin Nourizadeh ${ }^{1}$, Ali Hafezeqoran ${ }^{2}$, Elnaz Shafiee ${ }^{3}$, Seyyed Mahdi Vahid Pakdel $^{1^{*}}$

1. Post-graduate Student, Department of Prosthodontics, Faculty of Dentistry, Tabriz University of Medical Science, Tabriz, Iran

2. Assistant Professor, Department of Prosthodontics, Faculty of Dentistry, Tabriz University of Medical Science, Tabriz, Iran

3. Post-graduate Student, Department of Prosthodontics, Faculty of Dentistry, Islamic Azad University of Medical Science, Tehran, Iran

\begin{abstract}
Epidermoid cysts are rare congenital tumors of the central nervous system (CNS), histologically benign and slow- growing lesions. Their frequency among primitive intracranial tumors is about $1 \%$ and they account for $40 \%$ of all intracranial epidermoid cysts of the cerebellopontine angle (CPA); the most common symptom which brings about difficulties in oral rehabilitation of these patients is facial weakness which diversely affects impression processes and use of prosthesis. We report the oral rehabilitation of a patient with a cerebellopontine angle epidermoid cyst using neutral zone approach.
\end{abstract}

Key words: Soft tissue thickness; Facial reconstruction; Cone Beam Computed Tomography

\section{Introduction}

Epidermoid cysts are congenital, rare, slowgrowing, benign lesions of CNS that arise from ectopic inclusion of ectodermal cells during closure of the neural tube between the third and the fifth weeks of embryonic life (1-3). They have a central core of keratin proteins, desquamating cells and cholesterol, lined with a stratified squamous epithelium (4). They account for $1 \%$ of all intracranial tumors $(1,2$, $4)$; about $40 \%$ of them are located in the CPA, representing the third most frequent lesion after acoustic neuromas and meningiomas (1, 3). Clinically these tumors produce an insidious and protracted symptoms and signs with slow growth of the mass involving cranial nerves and cerebellar and brainstem structures. The onset of symptoms occurs between the second and fifth decades of life. Common presentations include a long history of tinnitus and hearing loss; vestibular symptoms are seldom seen, and occasionally, symptoms of trigeminal neuralgia,

\section{Corresponding author:}

Seyyed Mahdi Vahid Pakdel

Post-graduate Student, Department of Prosthodontics, Faculty of Dentistry, Tabriz University of Medical Science,

Tabriz, Iran

E-mail: og.smvp@gmail.com

Receive date: 2014-08-17| Accept date: 2014-09-01| Publish date: 2014-09-21

DOI: 10.7575/aiac.abcmed.15.03.01.15 
facial paresis or hemifacial spasm, headache, hydrocephalus and chemical or aseptic meningitis occur (5).

\section{Case Report}

A 46-year old woman with poor socioeconomic status with the chief-complaint of difficulty in chewing was referred to the postgraduate department of prosthodontics, Tabriz University of Medical Sciences, Tabriz, Iran. In examination of medical history, CNS problems were detected. The patient reported that she has been suffering from decreased discrimination, tinnitus, disequilibrium, and facial weakness for 6 years and she was consequently referred to a neurologist. Noncontrast brain CT scan revealed a spacious mass at the right CPA with a crusher effect on the cerebellum (Figure 1). Epidermoid cyst and meningioma were considered as the differential diagnoses. After thorough examination of signs and symptoms, the cerebellopontine angle epidermoid cyst was reported. The cyst was surgically removed. The surgical complications associated with oral rehabilitation were facial nerve paresthesia and muscle weakness. In examination of past dental history, all of her teeth were extracted because of dental caries 2 years ago and patient had not undergone any prosthetic treatments. In extraoral examination, facial asymmetry was obvious with the concave profile in lateral exam (Figure 2a). Because of facial nerve paresthesia, the asymmetry was intensified during smile (Figure 2b).

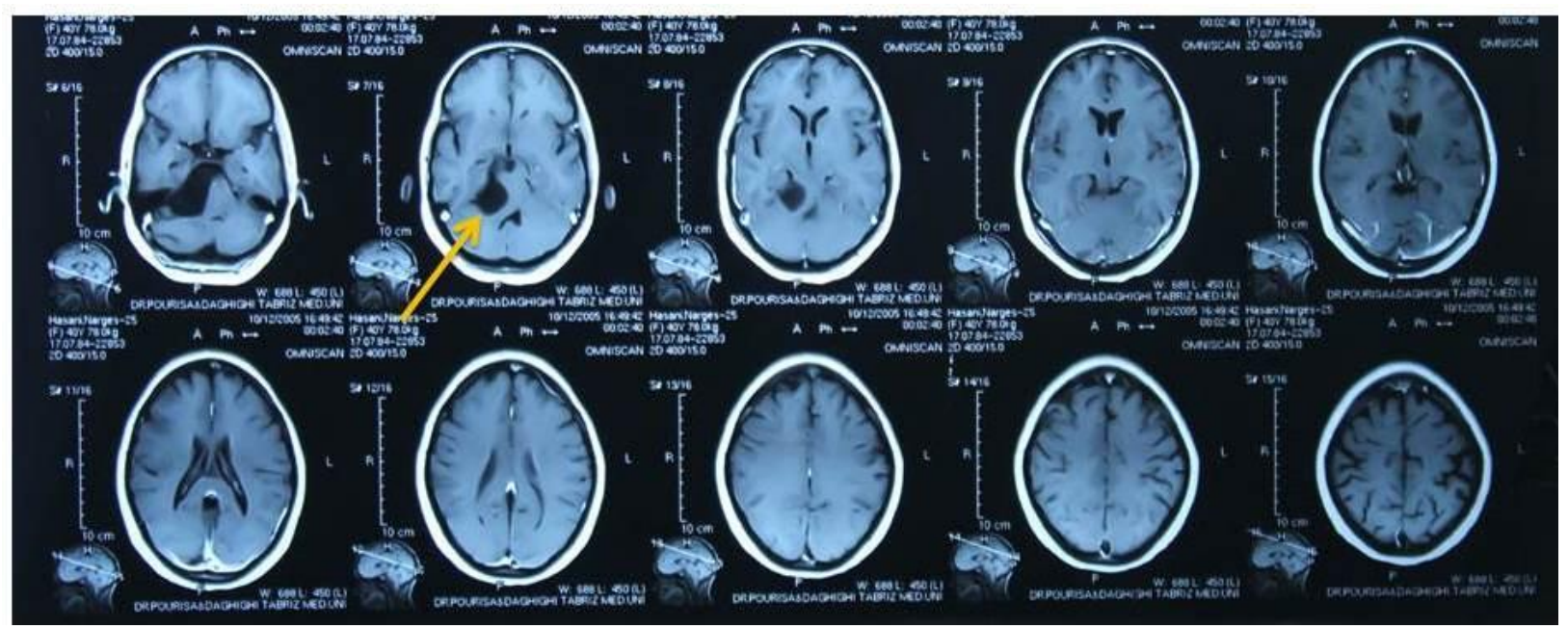

Figure 1: Non-contrast brain CT scan indicating a spacious mass at the right C.P angle (arrow) 

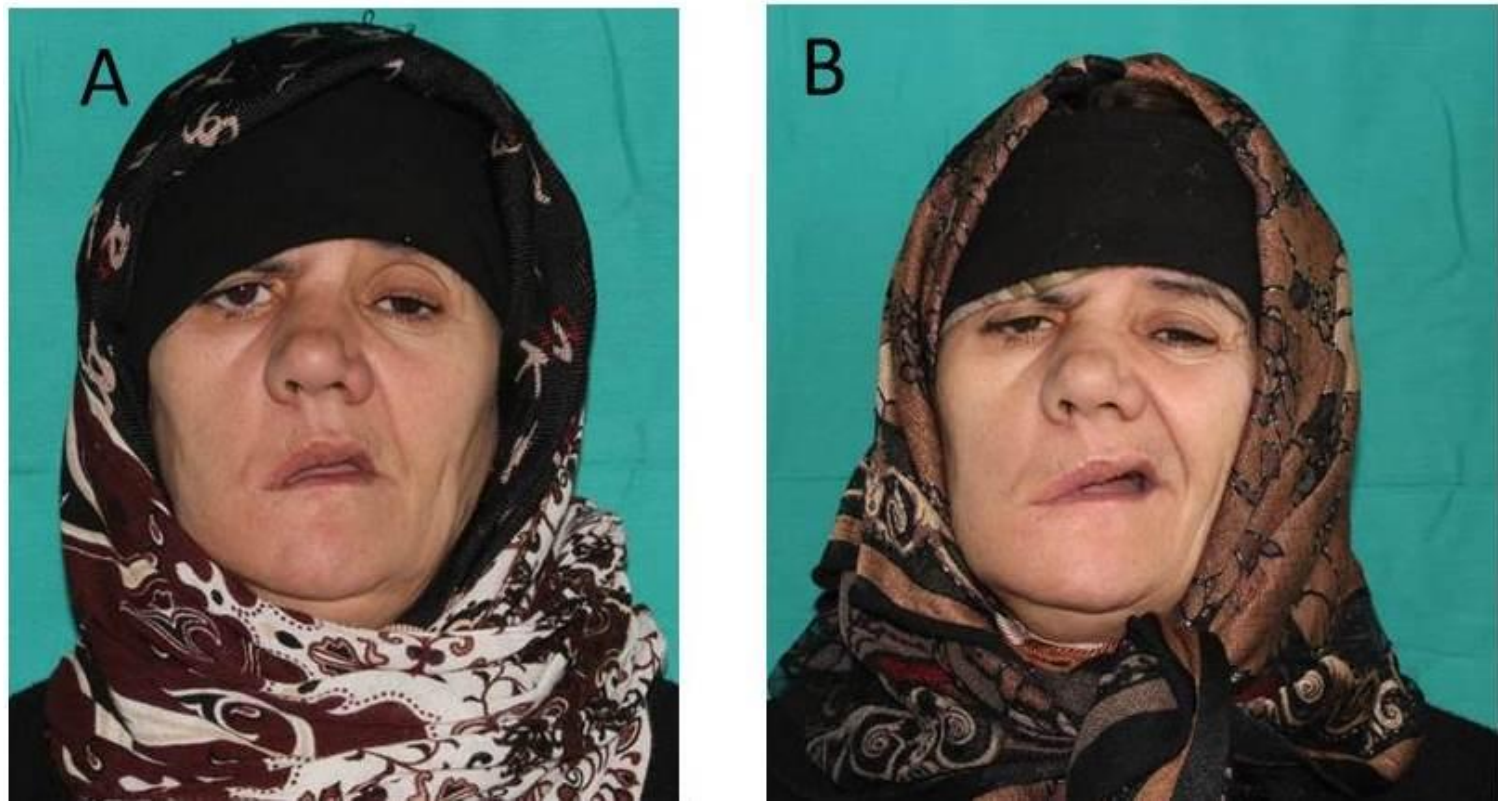

Figure 2: Frontal view of the patient at rest (a) and smile (b)

joints, decreased maximum opening was detected while deflection was taken place toward right side. Examination of masticatory
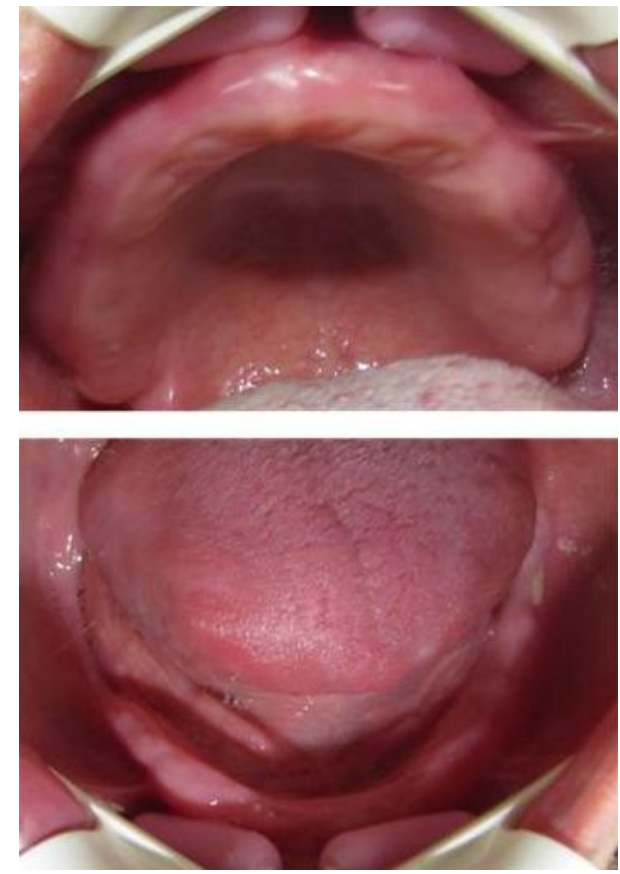

Figure 3: Maxillary and mandibular edentulous arches

Loose and asymmetrical upper lip was also obvious. In assessing temporomandibular muscles depicted looseness in right side. Intraoral examination indicated edentulous maxillary and mandibular ridges. Maxillary edentulous ridge was $U$-shaped and wellformed with highly-attached buccal frena. Mandibular ridge was moderately resorbed (Figure 3). Treatment options for oral rehabilitation were implant supported over denture or conventional complete denture. Since the patient had poor socio-economic status, conventional complete denture was administrated. For this purpose, primary impression was performed by compound and alginate wash. After fabrication of custom trays and border molding by low-fusing compound, final impression was made by zinc oxide eugenol paste (Cavex, Holland) (Figure 4). Later, temporary bases with wax rims were fabricated and adjusted in patient mouth according to anterior tooth show, inter-papillary line, alatragus line (for maxilla) and mouth corners and $2 / 3$ retromolar pad (for mandible). The vertical dimension of occlusion then was determined by physiologic and mechanical routes. Centric 
relation record was registered and after facebow recording, the master casts were mounted on a non-arcon semi-adjustable Hanau articulator. Neutral zone approach was employed for determination of the NZ dimensions. For this, wax rims were eliminated in posterior segments and replaced with selfcured acrylic resins with same height but narrower widths (Figure 5a). Tissue conditioner material (GC Soft, GC Inc., Japan) was added to buccal sides in both arches and low fusing (ISO) compound was added to lingual portions of mandibular arch. Later, the patient was asked to pronounce vowel sounds, do facial expressions in an exaggerated manner and perform deglutition. After finalization of NZ impression (Figure 5b), putty indices were
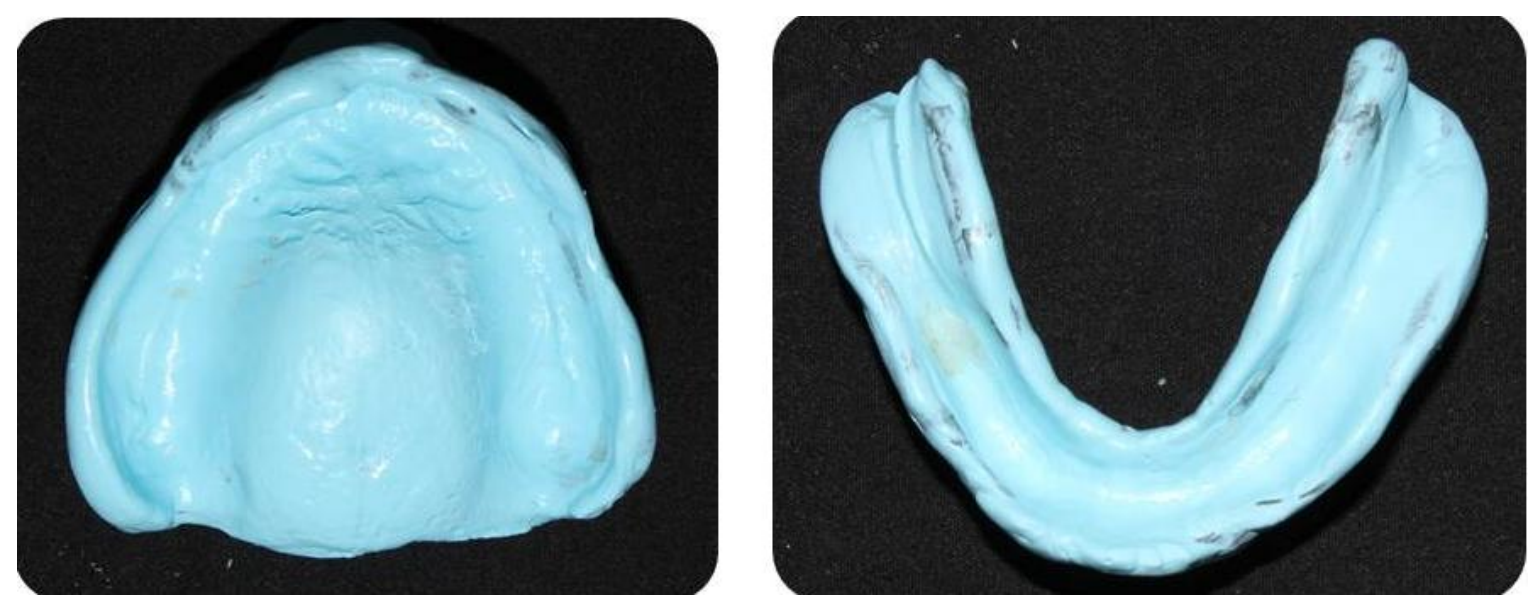

Figure 4: Final impression of edentulous arches made by ZOE paste

made for teeth arrangement guide (Figure 5c). Anterior and posterior teeth selection was made according to inter-alae distance and patient face configuration, and distance between canine and retromolar pad, respectively. Accordingly, Ivoclar acrylic teeth (Ivoclar, Vivadent, Liechtenstein) were selected: anterior, size 52 , shade A1; posterior, size N3, shade A1. After teeth arrangement in $\mathrm{NZ}$ indices (Figure $5 \mathrm{~d}$ ), teeth try-in was carried out and after processing, dentures were delivered to patient (Figure 6). Following delivery, hygiene instructions were addressed and scheduled follow-ups were performed. 


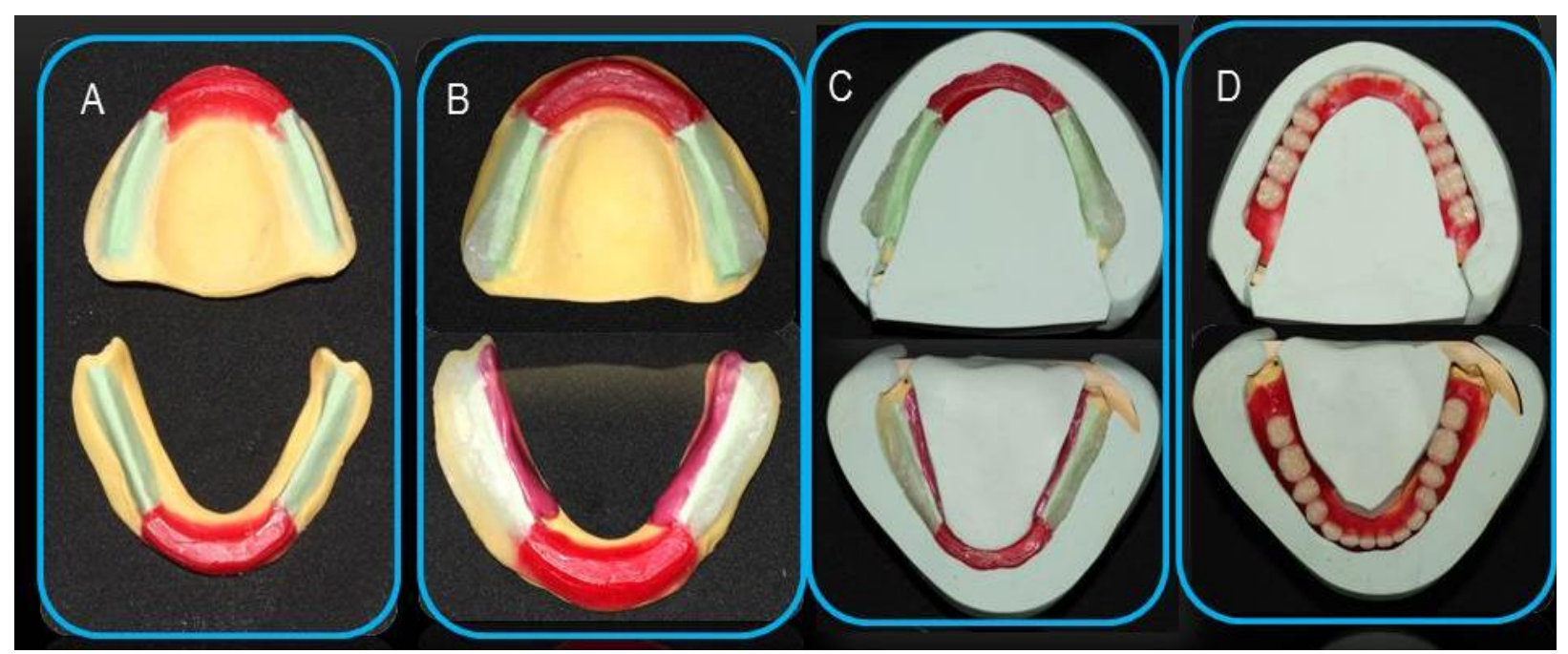

Figure 5: Base and rim preparation for neutral zone approach $(A)$, neutral zone impression (B), putty indices (C), and teeth arrangement in determined NZ (D)
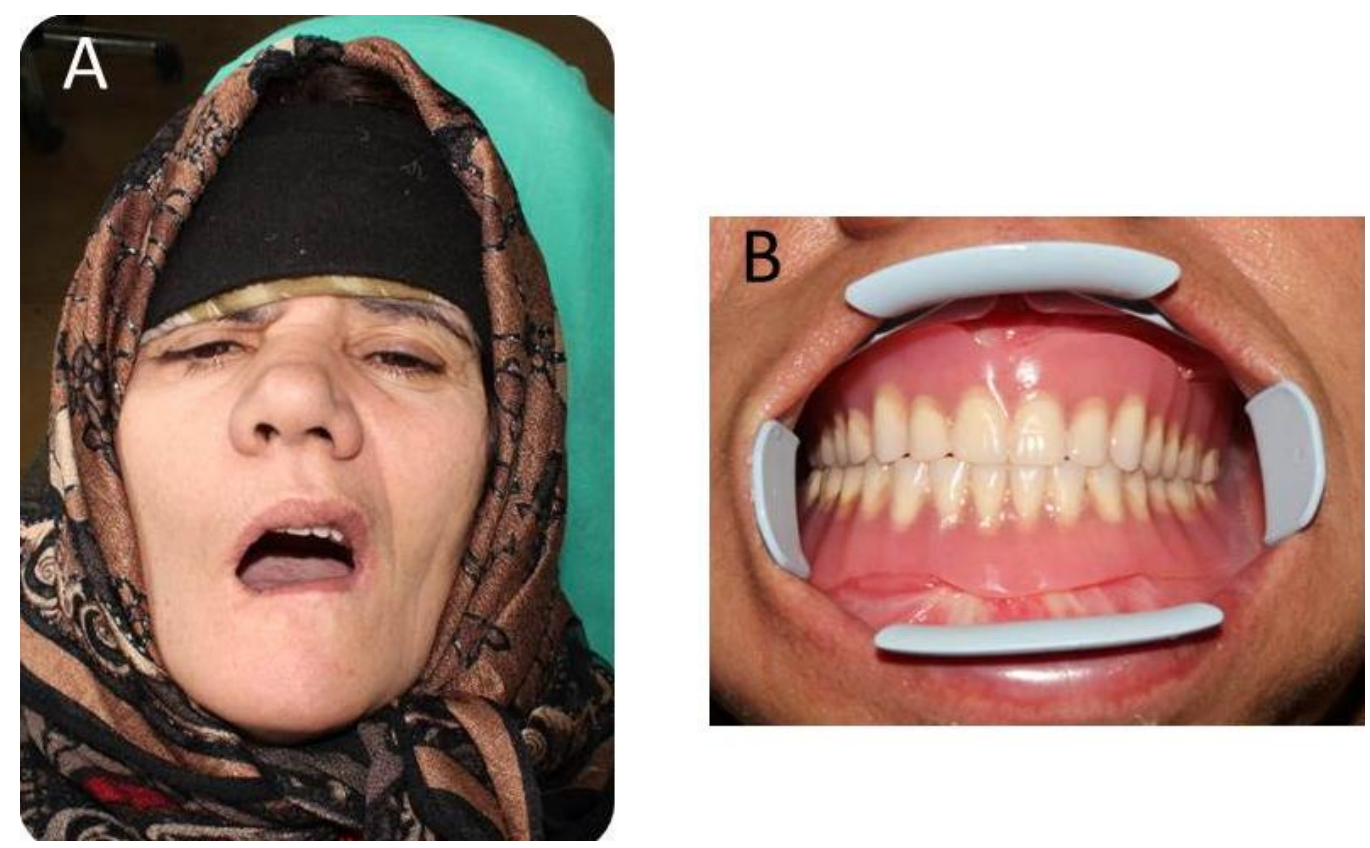

Figure 6: Teeth try-in (A) and denture delivery (B)

\section{Discussion}

Epidermoid cysts are benign tumors with slow growth that tend to envelop neurovascular structures without displacement $(6,7)$. Symptoms depend on compression of these structures; often they cause trigeminal neuralgia or facial paresis if fifth or seventh cranial nerves are involved. In other cases the eighth nerve involvement determines poor symptomatology because the slow growth does not cause vertigo, thanks to the compensation capability of the vestibular system. Audiological symptomatology can be poor. Indeed, our patient had an inconstant tinnitus in the side of the lesion with brief sensation of dizziness. This 
symptomatology and the absence of stapedius reflex only for a frequency required other tests such as $A B R$ and $M R I$ that established the diagnosis (8). However, normal caloric tests and cervical VEMPs showed the integrity of vestibule-ocular and sacculo-collic reflexes. The patient was controlled using MRI every 6 months, because the size of lesion and symptomatology did not suggest a surgical approach. The MRI allows to carefully check the exact growth rate of the lesion and to adopt the "wait-and-see" approach without any risk. This technique is essential during follow-up, especially when surgery is not the first and immediate choice, since MRI is highly specific and sensitive and avoids radiation for the patient $(9,10)$.

Although complete dentures are considered as mechanical devices in nature, they should be tailored for every patient to maintain the synchrony normal neuromuscular function. This would demand the proper functioning of all involved organs including the tongue, lips, cheeks, and floor of the mouth. This would assist maintaining oral functions such as speech, mastication, swallowing, smiling, and laughing. Unstable and unsatisfactory dentures could be the result of failing to distinguish the pivotal significance of tooth position and flange form and contour. The synchrony of complete dentures with neuromuscular function is the essential of any proper and successful dentures. The neutral zone is an area in the potential denture space where the forces of the tongue pressing outward are neutralized by forces of the cheeks and lips pressing inward. The magnitude and direction of these forces vary in in different individuals.(11, 12). In general, neutral zone technique is indicated when stability and patient's acceptance of lower CD are in question. Searching the literature revealed that this technique is found to be used in the following clinical situations: Severely atrophic mandibular ridge, Patients with prominent and highly attached mentalis muscle, lateral spreading of tongue as a result of poor transition from dentate to edentulous state and severe resorption, Patients with diminished neuromuscular control such as those with a history of stroke, Parkinson's disease or patients with impaired motor innervation to oral and facial muscles as a result of brain surgery, Patients with atypical shape or consistency of oral and perioral structures $(13,14)$.

\section{Conclusion}

This report addressed the oral rehabilitation of a patient with cerebellopontine angle epidermoid cyst for which impression making was carried out using neutral zone method.

\section{References:}

1. Son DW, Choi $\mathrm{CH}$, Cha SH. Epidermoid tumors in the cerebellopontine angle presenting with trigeminal neuralgia. Journal of Korean Neurosurgical Society. 2010;47(4):271-7.

2. Chu CK, Tseng HM, Young YH. Clinical presentation of posterior fossa epidermoid cysts. European archives of otorhino-laryngology : official journal of the European Federation of Oto-Rhino-Laryngological Societies. 2006;263(6):548-51.

3. David EA, Chen JM. Posterior fossa epidermoid cyst. Otology \& neurotology : official publication of the American Otological Society, American Neurotology Society [and] European Academy of Otology and Neurotology. 2003;24(4):699-700. 
4. Akhavan-Sigari R, Bellinzona M, Becker H, Samii M. Epidermoid cysts of the cerebellopontine angle with extension into the middle and anterior cranial fossae: surgical strategy and review of the literature. Acta neurochirurgica. 2007;149(4):429-32.

5. Dutt SN, Mirza S, Chavda SV, Irving RM. Radiologic differentiation of intracranial epidermoids from arachnoid cysts. Otology \& neurotology : official publication of the American Otological Society, American Neurotology Society [and] European Academy of Otology and Neurotology. 2002;23(1):84-92.

6. Safavi-Abbasi S, Di Rocco F, Bambakidis N, Talley MC, Gharabaghi A, Luedemann W, et al. Has management of epidermoid tumors of the cerebellopontine angle improved? A surgical synopsis of the past and present. Skull base : official journal of North American Skull Base Society [et al]. 2008;18(2):85-98.

7. Wang M, Li G, Jia D, Shen J. Clinical characteristics and surgical outcomes of patients with interdural epidermoid cyst of the cavernous sinus. Journal of clinical neuroscience : official journal of the Neurosurgical Society of Australasia. 2013;20(1):53-6.

8. Pont E, Mazon M, Ferrer AR. Cerebellopontine angle epidermoid cyst. Acta otorrinolaringologica espanola. 2013.

9. Di Rienzo L, Artuso A, Lauriello M, Coen Tirelli G. Pauci-symptomatic large epidermoid cyst of cerebellopontine angle: case report. Acta otorhinolaryngologica Italica : organo ufficiale della Societa italiana di otorinolaringologia e chirurgia cervico-facciale. 2004;24(2):92-6.

10. Mask-Bull L, Do B, Cattaneo JC. Brainstem epidermoid cyst in an individual with two previous intracranial epidermoid cysts: a rare diagnosis in a rare individual. Clinical neurology and neurosurgery. 2013;115(9):1888-90.

11. Beresin VE, Schiesser FJ. A study of the importance of the neutral zone in complete dentures. The Journal of prosthetic dentistry. 1991;66(5):718.

12. Beresin VE, Schiesser FJ. The neutral zone in complete dentures. The Journal of prosthetic dentistry. 1976;36(4):356-67.

13. Chandra SS. Management of a severely resorbed mandibular ridge with the neutral zone technique. Contemporary clinical dentistry. 2010;1(1):36-9.

14. Agarwal S, Gangadhar P, Ahmad N, Bhardwaj A. A simplified approach for recording neutral zone. Journal of Indian Prosthodontic Society. 2010;10(2):102-4. 